\title{
Assistência dos profissionais de saúde às crianças e adolescentes em situações de violência*
}

\author{
Rodrigo Jácob Moreira de Freitas ${ }^{1,2}$ \\ (1) https://orcid.org/0000-0002-5528-2995 \\ Natana Abreu de Moura ${ }^{1,3}$ \\ (D) https://orcid.org/0000- 0002-2517-9946 \\ Rúbia Mara Maia Feitosa ${ }^{1,4}$ \\ D https://orcid.org/0000-0001-7418-1156 \\ Deivson Wendell da Costa Lima ${ }^{5,6}$ \\ (D) https://orcid.org/0000-0002-7020-2172 \\ Livia Dayane Sousa Azevedo ${ }^{7}$ \\ (D) https://orcid.org/0000-0002-0019-9342 \\ Ana Ruth Macêdo Monteiro ${ }^{1,3}$ \\ (D) https://orcid.org/0000-0002-1130-1293
}

Artigo extraído de dissertação de mestrado "Práticas violentas vivenciadas por crianças e adolescentes em sofrimento psíquico: significados atribuídos pelos profissionais de saúde", apresentada à Universidade Estadual do Ceará, Fortaleza, CE, Brasil.

1 Universidade Estadual do Ceará, Fortaleza, CE, Brasil.

2 Universidade do Estado do Rio Grande do Norte, Faculdade de Enfermagem, Pau dos Ferros, RN, Brasil.

${ }^{3}$ Universidade Estadual do Ceará, Faculdade de Enfermagem, Fortaleza, CE, Brasil.

${ }^{4}$ Universidade Potiguar, Departamento de Enfermagem, Mossoró, RN, Brasil.

${ }^{5}$ Universidade de São Paulo, Escola de Enfermagem de Ribeirão Preto, Centro Colaborador da OPAS/OMS para o Desenvolvimento da Pesquisa em Enfermagem, Ribeirão Preto, SP, Brasil.

${ }^{6}$ Universidade do Estado do Rio Grande do Norte, Faculdade de Enfermagem, Mossoró, RN, Brasil.

7 Universidade de São Paulo, Faculdade de Medicina de Ribeirão Preto, Ribeirão Preto, SP, Brasil.
Objetivo: compreender a assistência realizada pelos profissionais de saúde às crianças e adolescentes que vivenciam situações de violência. Método: pesquisa fenomenológica por meio de entrevistas semiestruturadas, aplicadas de setembro a dezembro de 2015, com nove profissionais de saúde do Centro de Atenção Psicossocial Infantojuvenil da capital do Ceará, Brasil. As falas transcritas foram categorizadas e analisadas conforme o referencial teórico da Fenomenologia Social de Alfred Schütz. Resultados: os entrevistados realizaram consultas individuais e atividades de grupo para explorar os sentimentos, conflitos e percepções das vítimas. Além disso, assistiram a família e desenvolveram ações com profissionais de outros serviços em busca de resoluções das questões sociais dos casos de violência. Conclusão: os profissionais de saúde precisam conhecer as motivações e os significados que eles atribuem às suas experiências com vítimas de violência para embasar novas ações nos serviços de saúde.

Descritores: Assistência à Saúde; Pessoal de Saúde; Violência; Saúde Mental; Saúde da Criança; Saúde do Adolescente.

\section{Como citar este artigo}

Freitas RJM, Moura NA, Feitosa RMM, Lima DWC, Azevedo LDS, Monteiro ARM. Assistance of health professionals to children and adolescents in situations of violence. SMAD, Rev Eletrônica Saúde Mental Álcool Drog. 2020;16(1):1-8. doi: https://dx.doi.org/10.11606/issn.1806-6976.smad.2020.153963 


\title{
Assistance of health professionals to children and adolescents in situations of violence
}

\begin{abstract}
Objective: to understand the assistance provided by health professionals to children and adolescents who experience situations of violence. Method: phenomenological research through semistructured interviews applied in September to December 2015 with nine health professionals from the Child and Adolescent Psychosocial Care Center of the capital of Ceará, Brazil. The transcribed speeches were categorized and analyzed according to Alfred Schütz's theoretical framework of social phenomenology. Results: the interviewees carried out individual consultations and group activities to explore the feelings, conflicts, perceptions of the victims, in addition they assisted the family and developed actions with professionals of other services in search of resolutions of the social questions of the cases of violence. Conclusion: health professionals need to know the motivations and meanings they attribute to their experiences with victims of violence to support new actions in health services.
\end{abstract}

Descriptors: Delivery of Health Care; Health Personnel; Violence; Mental Health; Child Health; Adolescent Health.

\section{Asistencia de los profesionales de la salud a los niños y adolescentes en situaciones de violencia}

Objetivo: comprender la asistencia realizada por los profesionales de salud a los niños y adolescentes que experimentan situaciones de violencia. Método: investigación fenomenológica por medio de entrevistas semiestructuradas aplicadas en septiembre a diciembre de 2015 con nueve profesionales de salud del Centro de Atención Psicosocial Infantojuvenil de la capital de Ceará, Brasil. Las palabras transcritas fueron categorizadas y analizadas conforme al referencial teórico de la fenomenología social de Alfred Schütz. Resultados: los entrevistados realizaron consultas individuales y actividades de grupo para explorar los sentimientos, conflictos, percepciones de las víctimas, además asistieron a la familia y desarrollaron acciones con profesionales de otros servicios en busca de resoluciones de las cuestiones sociales de los casos de violencia. Conclusión: los profesionales de la salud necesitan conocer las motivaciones y los significados que ellos atribuyen sus experiencias con víctimas de violencia para basar nuevas acciones en los servicios de salud.

Descriptores: Prestación de Atención de Salud; Personal de Salud; Violencia; Salud Mental; Salud del Niño; Salud del Adolescente. 


\section{Introdução}

A violência é considerada um problema de saúde pública de ampla dimensão social, já que provoca um forte impacto na morbimortalidade das populações e aumenta a necessidade de atendimento em saúde das vítimas. Quando a violência é dirigida contra as crianças e adolescentes em sofrimento psíquico, ela repercute em traumas físicos, sexuais e emocionais que, apesar de não serem sempre fatais, são significativos potenciais de sofrimento, não apenas no momento da vivência da violência, mas ao longo de toda a vida(1-2).

Por sofrimento psíquico compreende-se tudo aquilo que afeta a ordem do sujeito, um conjunto de malestares e dificuldades de conviver com a multiplicidade contraditória de significados da vida relacionados às dificuldades de operar planos, definir o sentido da vida ou, ainda, relacionado ao sentimento de impotência e de vazio(3). Neste trabalho, será empregado o conceito, muitas vezes, como sinônimo de transtorno, adoecimento e problema mental.

Nesse contexto, os profissionais de saúde atuantes da rede de atenção à saúde, além de cuidar de pessoas com transtornos mentais, precisam ficar atentos às situações de violência nocivas para as crianças e os adolescentes, identificando os fatores protetores da violência e promovendo uma assistência interdisciplinar e intersetorial às vítimas. Para tanto, estas vítimas de violência necessitam ser acolhidas sem preconceito pelos profissionais nos serviços de saúde mental, sendo vistas como pessoas com condições singulares, únicas, e não apenas reduzidas ao seu diagnóstico e à medicalização excessiva(4).

$A$ assistência às crianças e adolescentes em situação de violência precisa ter cobertura ampla, integral e em rede, estabelecendo estratégias que considerem os problemas do território e as situações de violência vivenciadas e suas vulnerabilidades.

Este estudo visa a contribuir para a produção do conhecimento na área de saúde mental e violência, visto que, na literatura, a maioria das pesquisas ${ }^{(5-10)}$ enfatiza as consequências da violência na vida dessas pessoas, sem abordar a assistência realizada pelos profissionais de saúde frente à violência de crianças e adolescentes que já apresentam algum sofrimento psíquico.

Face a esta problemática, tem-se como objetivo compreender a assistência realizada pelos profissionais de saúde às crianças e adolescentes que vivenciam situações de violência.

\section{Referencial teórico-metodológico}

Este estudo é baseado no referencial teórico da Fenomenologia Social de Alfred Schütz, que se preocupa com as experiências humanas subjetivas, a fim de responder à pergunta: "O que ele quis dizer com essa ação no mundo da vida?". Assim, permite ao pesquisador aproximar-se das experiências significativas da pessoa ${ }^{(11)}$.

De acordo com o referencial adotado, toda ação é uma conduta humana concebida pelo ator, baseada em projeto preconcebido, e essa ação contém significado, podendo ser compreendida como significante quando se tem uma direção e, quando é direcionada a outros, se torna social. Assim, pode-se perceber a conduta dos profissionais como ações sociais no mundo da vida(11).

Essas ações possuem intencionalidade e são motivadas pelos interesses dos profissionais. Quando o motivo é o objetivo que se pretende alcançar com a ação, repercute-se em um ato projetado, e a situação vivida é previamente imaginada, sendo produzida uma ação futura, que se constitui na "motivação com-finalidade-de"(11).

A "motivação porque" faz alusão às experiências passadas, que levam a pessoa a agir como agiu, sendo o projeto a própria ação. Essas motivações têm íntima relação com a situação biográfica da pessoa e seu estoque de conhecimento à mão, que se refere às ações já realizadas pelos profissionais, possibilitando que eles reflitam sobre a própria experiência(11-12).

O encontro entre o pesquisador e profissional durante a entrevista permite estabelecer relação face a face e favorece a simultaneidade real de dois fluxos de consciência distintos e, somente assim, pode-se perceber o outro e conhecê-lo, captando a sua ação subjetivamente significativa no mundo da vida(11-12).

Assim, o encontro da entrevista exige, do pesquisador, um posicionamento de descentrar-se de si para se direcionar, intencionalmente, à compreensão da pessoa da pesquisa. Esse posicionamento denomina-se atitude fenomenológica. O uso da epoché, como atitude de redução fenomenológica da dúvida, é fundamental para se alcançar, ou se aproximar, da essência do fenômeno vivido(11).

\section{Método}

Trata-se de uma pesquisa fenomenológica desenvolvida no Centro de Atenção Psicossocial Infantojuvenil (CAPSi), no período de setembro a dezembro de 2015. O local da pesquisa foi escolhido por ser pioneiro no serviço público especializado em assistir a criança e o adolescente em sofrimento psíquico na capital do Ceará, Brasil. Além disso, este local da pesquisa possui relevância pela contribuição no processo de formação em saúde dos discentes de universidades.

Inicialmente, foram convidados 14 profissionais, mas dois se recusaram a participar da pesquisa e três não compareceram nos dias e horários agendados, totalizando nove participantes. A amostragem do estudo foi por acessibilidade. 
Os critérios de inclusão para a participação na pesquisa foram: ser profissional de saúde de nível superior com seis meses ou mais de tempo de atuação no CAPSi e ter realizado algum atendimento às crianças e/ou adolescentes em situação de violência. Excluíram-se aqueles que estavam afastados do trabalho devido a férias, licença de saúde ou maternidade.

A coleta de dados deu-se por meio da entrevista semiestruturada que teve a seguinte questão norteadora: como se dá sua assistência às crianças e adolescentes em situação de violência?

Durante a fase de coleta de dados, de acordo com o referencial teórico metodológico adotado, procedeu-se, simultaneamente, às etapas da análise dos $\operatorname{dados}^{(11)}$.

Após a organização do material colhido, as anotações dos relatores e transcrição das falas, foi lido o texto das transcrições das entrevistas individualmente, de forma minuciosa, a fim de se apropriar do material pesquisado. O material foi relacionado à questão norteadora da entrevista e as falas foram separadas em unidades de codificação, ou seja, os trechos mais representativos, que revelam os resultados mais significativos apontados pelos entrevistados. Em seguida, após a codificação individual das entrevistas, foram organizados e agrupados os significados apreendidos a partir das suas similaridades, consequentemente, definindo as categorias ${ }^{(13)}$.

As categorias evidenciadas foram descritas e discutidas, o que favoreceu um melhor entendimento sobre o fenômeno em estudo. Assim, os discursos foram interpretados para se chegar ao significado da ação das pessoas que foram analisadas, utilizando o referencial da Fenomenologia Social de Alfred Schütz, articulando-os com seus principais conceitos ${ }^{(11)}$.

A pesquisa foi submetida à avaliação e teve aprovação do Comitê de Ética e Pesquisa com o parecer 696.813, sob CAAE no 27056814.0.0000.5534. Foi designada a combinação letra e número, por exemplo, "E1, E2...", para preservar o anonimato dos participantes da pesquisa.

\section{Resultados}

Os participantes da pesquisa foram do gênero feminino, com faixa etária predominante de 25 a 30 anos, com média de idade de 35 anos. Elas trabalhavam no CAPSi há mais de um ano, desde a última seleção que ocorreu no município. Os profissionais de saúde foram: quatro psicólogas; duas terapeutas ocupacionais; uma enfermeira; uma fonoaudióloga e uma assistente social. Destas, apenas uma profissional estava cursando pós-graduação, nível mestrado, e as demais possuíam especialização, mas não na área de saúde mental. Nenhuma realizou cursos que abordassem a temática da violência.
A assistência dos profissionais de saúde à criança e ao adolescente em situação de violência foram divididas em três categorias: consultas individuais e atividades em grupo com as vítimas de violência; assistência à família das crianças e adolescentes em situação de violência; ações com profissionais de outros serviços de saúde e rede de apoio social.

Consultas individuais e atividades em grupo com as vítimas de violência

Os profissionais apontaram "motivações porque" nas consultas individuais e nas atividades em grupo, no enfrentamento dos conflitos e das frustrações relacionados à violência com a criança e adolescentes, pensadas como "motivos com-finalidade-de" evitar comportamentos violentos dos mesmos. Para o profissional do CAPSi, é importante que ele não enfoque apenas no problema mental, mas nas questões inerentes ao ser criança e adolescente.

A gente procura trabalhar questões de ser criança ou adolescente e não apenas sobre doença e a violência, isto também é importante. (E1); Eles falam, nas consultas, da dificuldade de se adaptar à escola, de ter relações sociais. O nosso trabalho mesmo é o de não olhar como o doente, mas fazer com que ela consiga se adaptar bem a esse contexto. (E4); Na atividade de grupo com crianças, a gente trabalha muito essa questão da criança ser frustrada, dos conflitos. Ela tem oportunidade de falar sobre o conflito e, nesse momento, a gente dá apoio. (E9)

A assistência à criança e/ou ao adolescente, seja individualmente ou em grupo, concerne ao trabalho das questões da personalidade e de socialização, ajudando a superar conflitos do cotidiano desses indivíduos. Dessa forma, os profissionais estarão promovendo ações para as pessoas do CAPSi que passaram por situações de violência, seja ela física, sexual, psicológica, bem como situações de exclusão social relacionadas ao estigma da doença mental que se deram em casa, na escola ou na própria comunidade, não apenas centradas no diagnóstico de saúde mental, mas também, prevenindo situações que, ao invés de ser resolvida com violência, poderão ser resolvidas com o diálogo e construção de vínculos.

Vale destacar que a unidade não possuía grupos específicos para trabalhar questões de violência vividas por esses sujeitos. A assistência acontece de maneira pontual à medida que os casos eram identificados.

Assistência à família das crianças e adolescentes em situação de violência

Nessa categoria, as "motivações porque" da assistência realizada pelos profissionais de saúde do CAPSi foram os atendimentos com os familiares das 
crianças e adolescentes em situação de violência. As "motivações com-finalidade-de" foram para que os familiares, em especial, as mães, possam desabafar sobre suas angústias.

Os grupos de família são voltados para esse suporte familiar que, muitas vezes, essas pessoas chegam aqui muito perdidas, muito confusas, não só com relação à doença, mas como lidar com aquelas crianças em sofrimento devido à violência. (E1); A gente percebe que esse não é um problema só da criança ou só do adolescente, é também direcionada à família. A família é que vai fazer o cuidado dessa criança. A gente sempre enfatiza isso nos grupos de família, nos grupos de mãe, a gente dá o espaço para elas colocarem as situações que estão vivendo. (E2); o grupo é um espaço de acolhimento para elas poderem compartilhar as experiências, contar uma com as outras, e aprenderem a lidar melhor com a situação, mas também para esquecer, ao mesmo tempo, um pouco o adoecimento do filho e pensarem um pouquinho nelas também. (E6)

A atividade de grupo com as famílias possibilitou o confronto de seus problemas e a reflexão sobre suas posturas para com a criança e o adolescente, visto que a relação entre mãe e filho foi fragilizada pela dificuldade dos membros em lidar com o problema mental. Com esse tipo de relação, a família passa a não cuidar da pessoa em sofrimento psíquico, configurando-se em uma situação de violência vivida por ela. Essa violência, por vezes, é expressa pela falta de paciência com a criança, buscando, na violência, uma forma de lidar com a situação.

Ações com profissionais da rede de atenção à saúde e de apoio social

As "motivações porque" de enfrentamento citadas nessa categoria abrangem a atenção em rede, articulada com profissionais de outros serviços de saúde e da rede de apoio social, e as "motivações com-finalidade-de" visam a uma atenção integral, que articule não somente o mental e a criança vítima dos diversos tipos de violência, mas, também, o físico, o social e a família.

É um trabalho em rede que a gente faz. Não só o atendimento individual e o grupo, mas também que essa criança tenha o suporte no CRAS [Centro de Referência de Assistência Social], no CREAS [Centro de Referência Especializado de Assistência Social], enfim, em outras instâncias e também em outras instituições que a gente possa estar fazendo parceria. (E3); Eu, pelo menos, não consigo desligar o mental do físico, vejo outras questões, como o social. Se o adolescente já tem uma capacidade de desenvolver uma tarefa que proporcione uma chance no mercado de trabalho, a gente busca essa estratégia, de olhar ele como um todo. (E8)

Os profissionais de saúde do CAPSi assumem o compromisso de olhar a pessoa em sua totalidade, construindo estratégias em conjunto com os diferentes profissionais que atuam nesse contexto. Embora haja essa necessidade e seja reconhecida a importância do trabalho em rede, os entrevistados apontam muitas dificuldades para que ela funcione, conforme expresso nas falas.

Eu escutei de uma profissional do CREAS: "às vezes, um caso que envolve saúde mental a gente não sabe o que fazer". (E5); É como se o paciente que tem transtorno mental fosse só o transtorno ou só vítima de violência. Não é considerado uma pessoa que acessa a rede de saúde por outras intercorrências que não a psiquiátrica. É como se restringisse; aí, é CAPSi; o CAPSi tem que resolver, e não é; é um cidadão, ele tem uma vida que envolve as outras dimensões. (E5); Essa articulação com outros profissionais tem alguns entraves. A maioria dos outros profissionais, eles também têm essa dificuldade. Material é escasso e eles têm pouca motivação. Acredito que, às vezes, falta motivação na gente também. (E7)

Além das dificuldades estruturais, apontou-se o desconhecimento dos outros setores quando se envolve um caso de violência a uma pessoa em sofrimento psíquico. Essa visão limitada sobre a pessoa restringe a atuação da rede de atenção em saúde e sobrecarrega o CAPSi.

\section{Discussão}

As políticas de saúde mental regem a assistência dos profissionais às pessoas em sofrimento psíquico, dentre estas, às crianças e aos adolescentes, inclusive, àqueles em situação de violência. Esta assistência, por meio de consultas individuais e atividades em grupos, assume uma função social, que precisa ir além do fazer o meramente técnico, com a inclusão do acolhimento e da escuta em busca de "dar voz" às pessoas envolvidas na violência(14).

Ao "dar voz" às pessoas envolvidas na violência, é possível contribuir para a implementação de ações efetivas frente a esse fenômeno, haja vista que ele repercute na maior participação da pessoa em sofrimento no tratamento, enfrentamento de estigmas e melhoria da qualidade de vida. Ao falarem sobre si e ao se identificarem com suas próprias histórias, a criança e o adolescente veem possibilidades de encontrar novos significados e novas formas de inserção na sociedade e na família(14).

A escuta é um instrumento terapêutico usado na consulta individual e nas atividades em grupo, que orienta para o Tu na relação face a face e, assim, efetiva a relação do Nós, em um movimento de reciprocidade motivacional. Essa relação recíproca gera uma série de mudanças nos significados subjetivos construídos no mundo intersubjetivo(11). 
A relação face a face é a orientação para o Tu, quando outra pessoa está ao alcance da experiência direta de outra pessoa, compartilhando um tempo e um espaço em comum. Ao estar presente pessoalmente, a pessoa está consciente da outra pessoa como ela própria, fluindo a sua experiência lado a lado e participando da relação do Nós intencionalmente conscientes de que assumiram uma orientação para o Tu ${ }^{(11)}$

Nesse cenário, a atuação dos profissionais de saúde nos grupos é fundamental para o convívio com a diversidade de pessoas que compartilham de experiências semelhantes. Assim, as atividades em grupo, desde que bem pensadas, em sua finalidade, estrutura e manejo clínico, permitem uma poderosa e rica troca de experiências e transformações subjetivas que não seria alcançável em um atendimento apenas individualizado. Isto se deve exatamente à diversidade de conhecimento e à pluralidade de seus integrantes ${ }^{(15)}$.

É na interação com os outros nas atividades em grupo que o eu reconhece a existência de outro eu semelhante, tendo os dois uma consciência similar, em que esse eu percebe que ambos fazem parte de um mundo exterior, o mundo da vida, reconhecendo-se como ser social, pois vivencia esse mundo com outros seres semelhantes. Considera-se essa interação nas atividades em grupo enquanto um intermediário da relação pessoa e sociedade no qual a pessoa passa a se reconhecer enquanto participante, produtor e produzido por essas relações com o coletivo(11).

A assistência de apoio institucional às vítimas de violência e às suas famílias, integrando o grupo familiar às práticas de acompanhamento terapêutico, tem sido apontada, também, como uma perspectiva pertinente na atenção à saúde dessas pessoas ${ }^{(16)}$.

A família caracteriza-se como um grupo existencial que compartilha com o indivíduo o seu sistema de tipificações e relevâncias. É um modelo básico de socialização que estabelece uma rede de relações com regras, costumes, valores e crenças. As modificações da estrutura familiar podem estar diretamente relacionadas à sociedade ${ }^{(11)}$.

A desestruturação familiar contribui para o agravamento do sofrimento das crianças e dos adolescentes. Esse sofrimento da família reflete no comportamento da criança e do adolescente, que precisa ser trabalhado por meio da construção de vínculo com a instituição, pois é nela que a família busca o apoio para cuidar de seus familiares em tratamento. É cuidando da família que o profissional de saúde do CAPSi estará cuidando da pessoa em sofrimento psíquico.

O compartilhamento das vivências entre os familiares nas atividades de grupo, organizado em forma de uma teia de apoio, é possível por meio de uma boa relação com os profissionais de saúde, onde se cria um sustentáculo de dificuldades e um arsenal de facilidades, com a percepção de alternativas de manejo clínico e reconhecimento de si no outro(11).

Raramente, os profissionais direcionam uma assistência aos familiares de crianças e adolescentes em sofrimento no CAPSi. Em geral, o foco é na pessoa em tratamento e/ou em algum membro individualmente, por exemplo, as mães, fragmentando, assim, as ações de apoio familiar. A existência da assistência a todos os familiares auxilia na percepção sobre o sofrimento psíquico das crianças e dos adolescentes em situação de violência e, por conseguinte, na busca de resolução com base em recursos disponíveis pela própria família(16-17).

A fenomenologia de Schütz pode contribuir para que o profissional tenha uma atitude de redução fenomenológica (epoché), descrevendo o mundo como se apresenta na consciência, seguindo em direção à essência do fenômeno. A compreensão desse conceito é fundamental para uma ação profissional sem julgamentos e discriminação com as famílias, crianças e adolescentes em sofrimento psíquico(11).

Outra categoria em destaque foram as ações dos profissionais do CAPSi com profissionais da rede de atenção à saúde e de apoio social nos casos de violência. As ações em rede potencializam uma atuação mais abrangente e multidisciplinar de um conjunto de atores de diversas instituições. A integração das instituições envolvidas na problemática da violência permite compartilhar conhecimentos e experiências, ampliar informações e resoluções ${ }^{(18)}$.

O contato com outros pares é necessário e significa vivência de envolvimentos interativos, com muitas pessoas, em complexas redes de relacionamento sociais. Há, a partir de encontros intersubjetivos, a construção intrínseca da situação biográfica de cada um, do que se é hoje, foi ontem e será amanhã, bem como a referências para a ação social(11).

Conhecer a rede de atenção à saúde e de apoio social disponível pode contribuir para o cuidado à família e às crianças e adolescentes, pois se constitui em possibilidades de ampliar estratégias para aliviar tensões e compartilhar responsabilidades ${ }^{(10)}$.

A assistência dos profissionais em rede significa compartilhar responsabilidades, promovendo o acompanhamento do caso até a sua inclusão e o seu atendimento em outro serviço, muito diferente de um procedimento administrativo e burocrático de preencher apenas uma guia de encaminhamento para outro serviço $^{(6)}$.

Também se compreende a assistência dos profissionais em rede como uma articulação política entre pares que, para se estabelecer, exige: reconhecer que o outro existe; conhecer o que o outro faz; colaborar, prestar ajuda quando necessário; cooperar, compartilhar 
saberes, ações e poderes e associar-se, compartilhar objetivos e projetos. Estas condições preliminares resultam, respectivamente, em autonomia, vontade, dinamismo, multiliderança, informação e múltiplos níveis de operacionalização(19).

O relato da ausência de comunicação entre estas instituições da rede, no estudo ora apresentado, representa uma denúncia por parte dos profissionais. É preciso ter clareza de que uma rede efetiva não representa um conjunto de instituições e profissionais que atuam isoladamente, mas, sim, instituições e profissionais que se reconheçam, tendo consciência da finalidade e do papel de cada instituição, para que o trabalho ocorra de forma horizontal e descentralizada, a fim de maior qualidade de informações e encaminhamentos.

\section{Conclusão}

Este estudo alerta para a necessidade de o profissional de saúde tomar conhecimento das suas motivações e dos significados que ele atribui às suas experiências na assistência às crianças e adolescentes em situação de violência.

A assistência à criança e ao adolescente em situação de violência perpassa pela subjetividade do profissional e de como o fenômeno da violência é significado por ele. Nas falas das pessoas da pesquisa, reconhecem-se as formas de enfrentamento dessa temática por meio da importância dada ao trabalho em grupo, do cuidado além do adoecimento mental, do apoio familiar, a tentativa do trabalho em rede, mas se aponta também a falta de dispositivos institucionais próprios e específicos para orientar melhor a ação profissional diante dos casos de violência que acometem os pacientes do serviço.

Este estudo possibilitou, aos profissionais de saúde, uma reflexão sobre sua assistência às crianças e aos adolescentes em situação de violência que, muitas vezes, não conseguem visualizar os seus "motivos porque" durante sua atuação no local de trabalho. Somente quando a assistência foi questionada ao profissional, ela tornou-se ato, pois ele resgatou as ações já realizadas, como observador de si próprio, e avaliou quais circunstâncias foram determinadas para que ele fizesse o que fez, na perspectiva de modificar sua ação futura. Uma atitude em epoché pode auxiliar o profissional, livrando-o de julgamentos e pré-conceitos sobre as crianças e adolescentes e suas famílias em situação de violência.

Como limitação da pesquisa, tem-se o fato de a coleta ter sido realizada em um único serviço de saúde mental e, nesse sentido, recomenda-se que mais pesquisas nesta perspectiva sejam realizadas devido à escassez de artigos publicados e, mais que isso: que essas pesquisas possam servir de "motivações com-finalidade-de", embasando ações da gestão e traduzindo-se efetivamente em ações nos serviços de saúde e de apoio social, contribuindo para o enfrentamento da violência.

\section{Referências}

1. Aragão AS, Ferriani MGC, Vendruscollo TS, Souza SL, Gomes R. Primary care nurses' approach to cases of violence against children. Rev. Latino-Am. Enfermagem. [Internet]. 2013;21(spe):172-9. [cited Jan 18 2016]. Available from: http://www.scielo.br/scielo.php?script=sci_ arttext\&pid=S0104-11692013000700022\&lng=en.

2. Fontes LFC, Conceição OC, Machado S. Childhood and adolescent sexual abuse, victim profile and its impacts on mental health. Ciênc Saúde Coletiva. [Internet]. 2017;22(9):2919-28. [cited Feb 17 2019]. Available from: http://www.scielo.br/scielo.php?script=sci_ arttext\&pid=S1413-81232017002902919\&Ing=en.

3. Silveira LC, Feitosa RMM, Palácio PDB. A escuta do sofrimento psíquico relacionado ao trabalho: contribuições da psicanálise para o cuidado em saúde. Psicol Rev. [Internet]. 2014;20(10):19-33. [cited Apr 4 2019]. Available from: http://pepsic.bvsalud.org/pdf/per/v20n1/ v20n1a03.pdf

4. Ministério da Saúde (BR). Secretaria de Atenção à Saúde. Departamento de Ações Programáticas Estratégicas. Caminhos para uma política de saúde mental infanto-juvenil. $2^{a}$ ed. Brasília: inistério da Saúde; 2005. 72 p.

5. Silva JT, Melo S. Violência infantil: atuação do psicólogo no processo de auxílio à criança. Rev Psicol Saúde e Debate. [Internet]. 2018;4(1):61-84. [cited Feb 17 2019]. Available from: https://doi.org/10.22289/2446922X.V4N1A4.

6. Macedo DM, Foschiera LN, Bordini TCPM, Habigzang LF, Koller SH. Systematic review of studies on reports of violence against children and adolescents in Brazil. Ciênc Saúde Coletiva. [Internet]. 2019;24(2):487-96. [cited Feb 17 2019]. Available from: http://www. scielo.br/scielo.php?script=sci_arttext\&pid=S141381232019000200487\&lng=en.

7. Costa DKG, Reichert LP, França JRFS, Collet N, Reichert APS. Concepctions and practices of healthcare professionals regarding intrafamily violence against children and adolescents. Trab Educ Saúde. [Internet]. 2015;13(suppl.2):79-95. [cited Feb 17 2019]. Available from: <http://www.scielo.br/scielo.php?script=sci_arttext \&pid=S1981-77462015000500079\&lng=en\&nrm=iso $>$.

8. Magalhães JRF, Gomes NP, Mota RS, Campos LM, Camargo CL, Andrade SR. Intra-family violence: experiences and perceptions of adolescents. Esc Anna Nery. [Internet]. 2017;21(1):e20170003. [cited Feb 17 2019]. Available from: http://www.scielo.br/scielo.php?script=sci_ arttext\&pid=S1414-81452017000100203\&Ing=en. 
9. Hildebrand NA, Celeri EHRV, Morcillo AM, Zanolli ML. Domestic violence and risk for mental health in childhood and adolescence. Psicol Reflex Crit. [Internet]. 2015;28(2):213-221. [cited Jan 18 2016]. Available from: <http://www.scielo.br/scielo.php?script=sci_arttext\& pid $=$ S0102-79722015000200213\&lng=en\&nrm =iso $>$.

10. Vicente JB, Higarashi IH, Furtado MCC. Mental disorder in childhood: family structure and their social relations. Esc Anna Nery. [Internet]. 2015;19(1):107-114. [cited Jan 18 2016]. Available from: http://www.scielo.br/scielo.php?script=sci_ arttext\&pid=S1414-81452015000100107\&Ing=en.

11. Schutz A. Fenomenologia e relações sociais. Rio de Janeiro: Vozes; 2012. 357 p.

12. Freitas RJM, Moura NA, Monteiro ARM. Violence against children/adolescents in psychic suffering and nursing care: reflections of social phenomenology. Rev Gaúcha Enferm. [Internet]. 2016;37(1): e52887. [cited Jun 22 2017]. Available from: http://www.scielo.br/scielo.php?script=sci_ arttext\&pid=S1983-14472016000100702\&Ing=en.

13. Flick U. Introdução à pesquisa qualitativa. $3^{a}$ ed. Porto Alegre: Artmed; 2009. 408 p.

14. Ministério da Saúde (BR). Conselho Nacional do Ministério Público. Secretaria de Atenção à Saúde. Departamento de Atenção Especializada e Temática. Coordenação-Geral de Saúde Mental, Álcool e Outras Drogas. Atenção psicossocial a crianças e adolescentes no SUS: tecendo redes para garantir direitos. Brasília: Editora do Ministério da Saúde; 2014. 60 p.

15. Ministério da Saúde (BR). Secretaria de Atenção à Saúde. Departamento de Atenção Básica. Departamento de Ações Programáticas Estratégicas. Cadernos de Atenção Básica n. 34. Brasília: Editora do Ministério da Saúde; 2013. 176 p.

16. Cezar PK, Arpini DM, Goetz ER. Registros de Notificação Compulsória de Violência Envolvendo Crianças e Adolescentes. Psicol Cienc Prof. [Internet]. 2017;37(2):432-45. [cited Feb 17 2019]. Available from: http://www.scielo.br/scielo.php?script=sci_arttext\& pid $=$ S1414-98932017000200432\&lng=en\&nrm=iso

17. Silva GB Júnior, Rolim ACA, Moreira GAR, Corrêa CRS, Vieira LJES. Identification and reporting of abuse of children and adolescents by family physicians in ceará. Trab Educ Saúde. [Internet]. 2017;15(2):469-84. [cited Feb 17 2019]. Available from: http://dx.doi.org/ 10.1590/1981-7746-sol00058
18. Macedo EOS, Conceição MIG. Psychosocial care for children and adolescents in situation of violence: the psychologist and the attention network. Pesqui Prát Psicosoc. [Internet]. 2017;12(1):129-46. [cited Feb 17 2019]. Available from: http://pepsic.bvsalud. org/scielo.php?script=sci_arttext\&pid=S1809-8908 2017000100010\&lng=pt\&nrm=iso.

19. Ministério da Saúde (BR). Secretaria de Atenção à Saúde. Departamento de Ações Programáticas Estratégicas. Linha de cuidado para a atenção integral à saúde de crianças, adolescentes e suas famílias em situação de violências: orientação para gestores e profissionais de saúde. Brasília: Ministério da Saúde; 2010. 104 p.

\section{Contribuição dos autores}

Concepção e desenho da pesquisa: Rodrigo Jácob Moreira de Freitas, Rubia Mara Maia Feitosa, Ana Ruth Macêdo Monteiro. Obtenção de dados: Rodrigo Jácob Moreira de Freitas, Natana Abreu de Moura, Rubia Mara Maia Feitosa. Análise e interpretação dos dados: Rodrigo Jácob Moreira de Freitas, Rubia Mara Maia Feitosa. Redação do manuscrito: Rodrigo Jácob Moreira de Freitas, Natana Abreu de Moura, Rubia Mara Maia Feitosa, Deivson Wendell da Costa Lima, Lívia Dayane Sousa Azevedo. Revisão crítica do manuscrito quanto ao conteúdo intelectual importante: Rodrigo Jácob Moreira de Freitas, Rubia Mara Maia Feitosa, Deivson Wendell da Costa Lima, Lívia Dayane Sousa Azevedo, Ana Ruth Macêdo Monteiro. Orientação: Ana Ruth Macêdo Monteiro.

Todos os autores aprovaram a versão final do texto.

Conflito de interesse: os autores declararam que não há conflito de interesse. 